\title{
La Pena de Muerte en Inglaterra
}

\author{
Por el Profesor H.H.A. Cooper M.A.LL.B. (1)
}

Después de una campaña de ciento sesenta años, la pena de muerte ha desaparecido, efectivamente, del sistema penal inglés. La ultima batalla, que una escritora ha llamado "la cruzada", se realizó en el campo familiar del Parlamento inglés al fin del año 1965. Por fin, los abolicionistas obtuvieron la victoria, casi completa. Porque la controversia continúa, todavía, en otros países del mundo y, aún cuando no exista garantía de que no resurja otra vez en Inglaterra, me parece que este momento es propicio para recordar el pasado y pensar en el futuro. Inglaterra no es el único país que abolió la pena de muerte. Muchos otros países europeos lo han hecho con buen éxito y menos agitación. El interés de lo que ha pasado en Inglaterra se basa en lo dilatado de la campaña y en la actitud de las opiniones de los antagonistas. Una observadora de la cruzada en la Gran Bretaña opinó que "seguramente la experiencia de un pueblo puede ayudar a otros para que eviten los obstáculos de acciones imprudentes y los tropiezos de rechazos legislativos". La experiencia de los ingleses demuestra una riqueza de ambos sucesos, bien documentados. Si los ingleses no fueron los pioneros, seguramente la historia de sus esfuerzos es la más interesante.

En los primeros años del siglo XVIII, más de doscientos delitos llevaban como sanción la pena de muerte. El derribar un árbol, el matar una vaca ajena llevaban este castigo. También lo llevaban el delito de dañar uno de los puentes de Londres así como ciertos robos de propiedad de pequeño valor y el pedir limosna por parte de miembros de las fuerzas armadas. Estas penas no eran castigos en el papel. En 1808 un niño de siete años de edad y su hermana de once años sufrieron la pena de muerte. En 1814 se ahorcó a un hombre que derribó un árbol, que no era suyo. En 1833 otro niño de nueve años de edad sufrió la pena de muerte por el robo de pinturas valorizadas en dos peniques. Además, hasta el año 1868 todos los ahorcamientos eran espectáculos públicos y los días en que se realizaban estos repugnantes acontecimientos se consideraban como fiestas. La literatura inglesa contiene muchas descripciones espantosas del temperamento de la multitud en estas ocasiones. El gran novelista Thackeray, que fué

(1) Catedrático Visitante de Derecho Constitucional en la Universidad Nacional Mayor de: San Marcos. 
testigo de esto una vez, escribió acerca de la vergüenza que sintió a cousa. de su asistencia como parte del público.

¿Qué pensaban los grandes políticos y juristas del país acerca de estos castigos tan bárbaros? Felizmente, tenemos buenos relatos de sus palabras sobre estos temas. Son muy instructivas de la mentalidad de la época. Sus pensamientos no revelan ninguna vergüenza a causa de la crueldad de estas penas tan severas comparadas con la gravedad de los delitos. Un gran juez, jefe de la corte en su época, sentenciando a un pequeño sujeto de diez años dijo:

"El es sujeto apropiado para la pena de muerte $y$ debe sufrirla. Aunque quitar la vida de un niño de diez años parezca cruel es posible que el ejemplo de su castigo pueda disuadir a otros niños de cometer iguales delitos. Ademós, si se perdonase $\alpha$ este niño solamente a causa de sus años,. es posible que este hecho produciría una tendencia contraria $y$, por eso en bien de la sociedad la pena debe aplicarse".

Consecuentemente, el niño sufrió la pena, suspendido de una soga ante el público en su décimo año como ejemplo de perversidad $y$ para intimidar a les otros de manera que eludan el mismo fin.

Otra vez, un Lord Canciller, cuyo conocimiento de las legalidades del sistema inglés era profundo, dijo:

"Indudablemente, parece un infortunio que una pena tan pesada como la de muerte deba aplicarse a quién derriba un sólo árbol, o al que mate o al que hiera una vaca ajenos. Sin embargo, si fuese abolida tal pena, una persona podría arrancar o derribar campos de plantaciones o destruir todo el ganado de un hacendado sin sufrir la pena de muerte". Hay una gran cantidad de opiniones emitidas con el mismo objeto. $Y$ no se debe imaginar que las ideas expresadas vienen siempre de los más grandes reaccionarios de la época. El famoso filósofo, John Stuart Mill, cuyas opiniones políticas influyeron mucho en la formación de la doctrina del partido liberal, era un retencionista. Es un hecho notable, sin embargo, que ninguno de los líderes de los grandes partidos del siglo XIX se expresaba sobre esta cuestión. En particular, es interesante que los dos líderes más influyentes en la política inglesa en la ultima parte del siglo, el líder de los conservadores, Disraeli, y el líder del partido liberal, Gladstone, se mantuvieron en una posición de neutralidad frente a este problema.

Entre el comienzo del siglo XIX y el año 1868 hubieron muchos cambios sociales, económicos y políticos en la Gran Bretaña. En el primer año del siglo, el censo reveló una población de 14 millones de habitantes en todo el país, en el que la maquinaria y la factoria empezaron ya a cambiar la econornía de la nación. En lugar de riqueza natural, basada en la propiedad de grandes terrenos, la revolución industrial producía una clase, cuya riqueza se encontraba en la fabricación y en el mundo mercantil. No desapareció la pobreza ni tampoco mejoró mucho la vida de la clase baja. La miseria cambió, meramente, su naturaleza. Estos primeros años eran testigọs de una lucha entre las clases más ricas por el poder del Eistado. Poco a poco el valor del pueblo en general se impuso y los antagonistas principales exigieron, de vez en cuando, el apoyo de ciertas clases de la gente inferior. El año 1832 vió la reforma del sufragio que dió a muchas personas el derecho de elegir en forma más democrática sus representan- 
tes en el Parlamento inglés. En 1825, después de 25 años de represión total, el derecho de asociación libre se acordó a los sindicatos. A mediados del siglo el movimiento reformista se aceleró y por el año 1867 la mayor parte de los hombres de más de 21 años de edad tuvo el derecho de votar. El efecto de esta nueva democracia es bien clara en el campo del derecho penal.

Había, durante el siglo XIX, muchas comisiones que estudiaban el problema de la pena de muerte. En el año 1861 la pena de muerte quedó sólo para casos de traición o asesinatos del tipo más grave, el crimen que se llama en inglés "Murder". Además, en el año 1868 se prohibió todos los ahorcamientos públicos. Nadie puede discutir que esta mitigación en la penalidad no se relacione con los cambios políticos y el ambiente favorable a la clase baja, producido por éstos. Sin embargo, un grupo de reformadores no quedaron satisfechos aún por este mejoramiento. Estas personas persistieron en sus esfuerzos hacia la abolición total de la pena de muerte. En Inglaterra, durante el resto del siglo y los primeros años del presente, este movimiento no tenía mucha fuerza. Mientras tanto, cinco Estados de los Estados Unidos de Norte América abolieron la pena de muerte y también abrogaron sus leyes en este sentido los países Europeos de Bélgica, Holanda y Portugal. Sin abrogación formal sino con el mismo efecto la pena de muerte no se impuso en Dinarmarca desde 1892. La cruzada en la Gran Bretaña continuaba y en el año 1930 cobraba velocidad. Desgraciadamente, la cuestión cayó en la política confusa de la época anterior a la segunda guerra mundial. Después de la victoria y con la acensión nuevamente de un gobierno laborista, se reanudó la lucha de los abolicionistas. No es posible describrir los acontecimientos de los últimos años de la campaña, sin hacer mención del trabajo de Roy Calvert. Cuando tenía 27 años de edad, Calvert, renunció a un puesto seguro en el Servicio Civil del Estado para dedicarse a la cruzada. Debido a su trabajo y principalmente como resultado de la publicación de su gran libro el movimiento abolicionista asumió una base racional que dió dirección $Y$ fuerza a la campaña dentro del Parlamento. Como parte del Estado Socialista que surgió después de la guerra se impuso una moratoria en 1948. Su efecto era la suspensión de la pena de muerte por un plazo experimental de cinco años. El año 1952 produjo una reacción contra el movimiento izquierdista en Inglaterra y en ese año volvió al poder un gobierno conservador, cuyos partidarios estaban dedicados en gran parte a la reintroducción de la pena de muerte por asesinatos y el aumento general en la severidad de los castigos por otros delitos. Sin embargo, el gobierno, reconociendo la inquietud pública frente a esta cuestión, esperó el informe de una Comisión Real nombrada en 1949 por el gobierno laborista. La tarea de esta Comisión se restringió a la consideración de la cuestión de una modificación en la ley referente a la pena de muerte. Se excluyó, expresamente, y algunos dirían curiosamente, la cuestión de la abolición total de esta pena. El informe de la Comisión salió en 1953. Era un documento formidable. La Comisión tra. bajó muy diligentemente y recibió evidencias y relaciones de muchos países del mundo. Por eso, el informe de esta Comisión es esencial para cualquier estudiante del problema. Y a pesar de las limitaciones, la Comisión concluyó que lo único importante era la abolición o retención de la pena 
de muerte, reconociendo la elección entre esa alternativa como una cosa política. El gobierno conservador se enfrentó a un dilema. Sus partidarios demandaron la reimposición de la pena de muerte ante la ola creciente de crímenes producidos por una sociedad próspera, cuya moralidad estaba en estado de cambio. Consecuentemente, no renovó la moratoria y la pena de muerte se reimpuso de nuevo para todos los asesinatos indiferentes a su carácter. Después de un lapso de cuatro años se promulgó una medida en 1957 que era una componenda no satisfactoria. La ley estableció dos categorías de asesinatos, la primera llevando la pena de muerte y la otra el castigo de cárcel de por vida. La diferenciación es curiosa y no muy lógica. Se reservó la pena capital para asesinatos cometidos con armas de fuego, en el transcurso de un robo, durante un escape o rescaie de preso. Además la impuso en el caso de asesinar a un agente de policía o para asesinatos múltiples. Entonces, el que matara a un sujeto con una hacha o por puñaladas no sufría la pena de muerte. El envenenador más temible escaparía a la pena capital si seleccionara, cuidadosamente, su víctima $y$ restringiera su repugnante apetito. Durante el largo periodo del poder de los conservadores esta situación permaneció, $\alpha$ pesar de los esfuerzos de un decidido grupo de individuos de ambos partidos, dedicándose $\alpha$ la abolición total de la pena de muerte. Volvió a ser una cosa política, con el partido laborista en favor de la abolición y el gobierno conservador igualmente opuesto a cualquier cambio de este carácter. En 1964, el gobierno conservador cayó y luego los abolicionistas, casi sin la ayuda oficial del nuevo gobierno laborista, obtuvieron la promulgación de una ley que ha suspendido la imposición de la pena de muerte en todos los casos de asesinatos por un plazo experimental de cinco años. Quedan vigentes ciertas leyes que prescriben la pena capital en casos de traición y el incendio de ciertos diques navales, pero estos delitos están casi caducos. Pues se puede decir que Inglaterra después de 165 años ha abolido efectivamente la pena de muerte y ha eradicado lo que los abolicionistas consideraban una mancha en nuestra jurisprudencia.

Tengo que mencionar algo pertinente que tenía importancia especial en el caso de Inglaterra. El monarca ha tenido siempre y sigue teniendo una reserva de poder indefinida que se designa como la prerrogativa real. Incluye el poder de perdonar y aliviar la miseria de súbditos condenados a la pena de muerte, sustituyendo otra pena menos severa, en la práctica, la pena de cárcel de por vida. Bajo la constitución británica este poder se - ejerció según los consejos de los ministros del Estado y el Ministro responsable en este caso es el Ministro de Gobierno. El recibía las solicitudes de los condenados, pidiendo la merced del soberano y él tenía que decidir si estos súbditos deberían ser o no ahorcados. El ejercicio de este poder casi juticial por un ministro tan politizado, produjo muchas inquietudes en los últimos años e impuso un deber insoportable en algunos Ministros con buena conciencia y fuertes sentimientos sobre este asunto. El efecto, sin embargo sirvió, para crear otras anomalías. De un número de más o menos cien sujetos condenados a la pena de muerte cada año, aproximadamente noventa por ciento hubieran recibido la mitigación de su sentencia. La controversia se refirió a las atras personas no perdonadas que sufrieron la pena capital. 
Esta es la parte histórica. Vamos a considerar sus lecciones. El Lord Canciller actual de Inglaterra, jefe de la judicatura y un decidido abolicionista, escribió un libro en el que clasificó los dos argumentos de los retencionistas como el racional y el emocional. Seguramente, esta clasificación servirá para analizar los argumentos opuestos de los abolicionistas. De los. puntos racionales examinados por Lord Gardiner sólo dos me parece que tengan relevancia er el contexto de esta exposición. El primer argumento en favor de la retención de la pena de muerte depende de la premisa de que esta pena es intimidatoria hasta un mayor límite que cualquier otro castigo. La evidencia contra esta creencia es abrumadora. Quizás la mejor evidencia se encuentra en el hecho de que en cualquiera de los países en que ha habido la abolición de la pena de muerte, los delitos que le correspondían no han aumentado. Existen en los Estados Unidos de Norte Ámérica, estados vecinos, uno abolicionista $y$ otro retencionista. Las estadísticas indican casi sin variación que ni la retención ni la abolición se refleja en la prevención de delitos. Como factor humano, considérese el hecho que en Inglaterra, durante el siglo XIX, de 167 sujetos ahorcados durante un plazo determinado, 163 vieron, personalmente, el ahorcamiento en público de un delincuente. Es obvio que en este caso no había ningún efecto intimidatorio. En los últimos años hubo un caso en que un joven violento mato $\alpha$ un empleado de un banco, seleccionando una arma de fuego, un arma que agravó el delito, a la misma hora en que se ahorcó a un sujeto, cuyo ahorcạmiento recibió la más grande publicidad. ¿Cómo se puede explicar el gran aumento en el empleo de las armas de fuego por asesinos a pesar del peligro introducido por esa ley irracional de 1957? Si se investiga cuidadosamente la materia, no se podría encontrar ninguna prueba que indique que las medidas severas tengan efecto verdaderamente intimidatorio. Sin embargo $\epsilon$ l argumento del gran filósofo Mill tiene validez. Dijo que mientras son obvios aquellos que no se han intimidado mediante la pena de - muerte ¿Cómo podemos asegurarnos de la cantidad de personas que pudieran haber cometido tal delito si no estuviese la pena capital como un desafío a su conducta? Claro es que no podemos conocer a estas personas y ningún método científico las revelaría. Pero en la práctica, la prueba es más fácil que lo que sugiere la complejidad del problema filosófico. Si el factor impartante es la presencia o ausencia de la pena de muerte, su abolición debería causar un cambio significativo en las estadísticas penales. En nuestros días la ciencia estadística ha venido a ser suficientemente exacta para buscar con confianza un aumento en los homicidios producidos por la abolición de la pena capital. Es cierto que en ningún país en que se han hecho tales estudios se ha notado tal aumento. La posición, entonces, es que es imposible probar, como ha dicho Mill, quienes hayan estado intimidados por la pena y por lo tanto no es posible ofrecer ninguna evidencia positiva del efecto intimidatorio de la pena basada en hechos. Y ninguna creencia tiene valor en la formación de una actitud científica frente a esta cuestión; sólo cuentan los hechos. Contra esta vaguedad hay hechos indiscutibles que señalan los casos en que la pena no ha tenido ningún efecto en sentido intimidatorio. No ha habido ningún argumento en favor de la retención de la pena de muerte basado en hechos; ni tampoco podría fa- 
bricarse. Juzgando entre los dos lados ¿Qué argumento, el retencionista o el abolicionista, debe atraer al hombre racional?

El segundo argumento puesto y analizado por Lord Gardiner es que no existe ningún castigo, en caso de asesinatos, adecuado o sastifactorio como alternativo. Esta discusión ha preocupado a los reformistas ingleses durante la historia entera del movimiento abolicionista. Esta cuestión toca el ámbito y filosofía del castigo. La única pena alternativa es la pena do córcel y en caso de delitos tan graves como asesinatos tiene que durar de por vida. No obstante que la penología inglesa no ha aceptado, todavía, la idea de la sentencia indeterminada, esta pena de por vida en caso de asesinatos tiene alguna semejanza. En primer lugar, el plazo de encarcelamiento nunca es, literalmente, de por vida; en el hecho la mayor parte de los asesinos condenados en Inglaterra han sido puestos en libertad después de nueve años y raras veces han sufrido detención por más de quince años. Naturalmente, existe el riesgo de que el criminal pueda repetir su crimen, pero la experiencia en Inglaterra y otros paises ha enseñado que este riesgo es mínimo.

Sin umbargo, a pesar de todo, alguien puede decir con cierta razón ¿Porqué hacemos tales cosas tan costosas cuando el remedio de la liquidación barata e indolorosa es mucho más fácil? La ejecución de estos inútiles delincuentes dejaría limpia la sociedad, evitaría todos los problemas de gastos y posibilidades de fugas o la repetición del delito. $\vec{A}$ primera vista tal proposición, en su simplicidad, es muy atrayente. Fay que admitir que no $\in$ s posible encontrar su refutación en el campo penológico. Este punto tiene una validez, pero su último valor se prueba según las normas de la filosofía del derecho y la experiencia de la vida política. ¿Quién va a ser árbitro de lo que debe ser criterio para aplicar la pena de muerte? Las preferencias son infinitas. La filosofía predominante en Inglaterra hasta la segunda parte del siglo XIX favoreció la imposición de la pena capital en casi cada instancia donde había una interferencia indebida a la propiedad. Fué una filosofía muy capitalista y podemos calcular sus resultados por la miseria humana que causó. O sea que se reserva para liquidar $\alpha$ sus enemigos políticos sobre la base de que estas personas constituyen una amenaza a la seguridad del Estado. Todas son buenas razones según la filosofía predominante. Sin embargo, todas son, sencillamente, expresiones de una enseñanza del poder para eliminar algo indeseable. Este argumento se rechazó, prudentemente, en mi concepto, por la Comisión Real inglesa de 1949/53. Como escribió Lord Gardiner esta es una cuestión ética Y las palabras de la Comisión merecen repetirse.

"Recientemente, se ha hecho la sugerencia a veces que el asesino insano debe ser castigado igualmente como el sano 0 , aunque no deba ser ahorcado como pena, se debe exterminarle indolorosamente como medida de higiene social. El argumento en cada instancia es igual su existencia continuada no será provechosa a él mismo y quedará no solamente como un lastre inútil sobre la comunidad sino como un riesgo porencial si existiera siempre la posibilidad de que pueda escapar y cometer otro delito. Tales doctrinas se han defendido y practicado en la Alemania Nacional-Socialista, pero son repugnantes a las tradiciones 
morales de la civilización occidental y tenemos confianza en que se las rechazaría por la mayor parte de la población de este país."

Nadie debe añadir más y para refutarlo es necesario buscar sus principios en otra filosofía u otra ética.

Todos los otros argumentos en pro o en contra son emocionales. El espacio no me permite tocar a estos, pero no tiene importancia en la formación de mi última posición. Antes de señalarla, quiero tratar sólo un punto que, a veces, confunde la cuestión. Hay personas, todavía, que basan sus objeciones contra la pena de muerte en el argumento de que ningún ser humano y ninguna agrupación de personas como el Estado tiene el derecho de tomar la vida de otra. Este argumento es, puramente, emocional $y$, en mi concepto, no tiene ninguna validez jurídica. Me declaro, sin temor, positivista y cualquiera que sea la posición moral, no hay ningún impedimento jurídico contra el Estado. Si el Estado no tuviera el derecho de tomar la vida, no tendría, tampoco, derecho de imponer multas, penas de cárcel o cualquier otro castigo. Sólo un anarquista puede discutir lógicamente esta cuestión desde esa premisa.

Soy abolicionista porque años de estudios no me han convencido de la utilidad o eficacia de la retención de la pena de muerte como castigo intimidatorio. Cuando alguien me ofrezca un argumento racional, basado en claras evidencias, en el sentido de que esta pena tiene más valor preventivo que otras medidas, yo estaré convencido de la necesidad para un cambio en mi punto de vista. Como filosofía penológica la idea de la higiene social no me atrae; temo que tal vez las opiniones de mis dirigentes no coincidan con las mías y entonces me encontraria en medio de un proceso de limpieza no tanto a mi gusto. Reconozco, francamente, que la cuestión es sólo parte de un tema más grande que toca a los fundamentos del sistema entero de castigos. Sin embargo, para mí, cualquier forma que tal sistema futuro y utópico tenga, la pena de muerte no encontrará ningún sitio dentro de su estructura.

Y asi continúa la cruzada. Hay países que retienen la pena de muerte $Y$, generalmente, estos países creen que la severidad de la pena se refleja en la disminución de los delitos. Las estadísticas y estudios serios pronostican un desengaño en cuanto a estas esperanzas. Ni siquiera en Inglaterra la victoria de los abolicionistas es tan segura que pueda poner a salvo las armas. Recientemente en Inglaterra hubo un juicio sensacional contra una pareja de jóvenes a quienes se han llamado "los amantes diabólicos". Tan espantosos fueron sus crímenes que produjeron como reacción una fuerte demanda para la reintroducción de la pena de muerte. Todo era pura emoción. Nada es mós ilustrativo de la futilidad penológica de este castigo que el hecho de que cuando estos monstruos cometieron sus asesinatos la pena de muerte tenía completa vigencia. Es cierto que la posibilidad de sufrirla no intimidó a estos sujetos. ¿Qué vale una pena que intimide a los buenos, mientras los malos la pasan por encima? 
1. "The Crusade against Capital Punishment" por Elizabeth Oman Tuttle. (1961) Stevens, London.

2. "Capital Punishment in the Twentieth Century" 5th edn. por Eric Roy. Calvert, (1936) Putnam, London.

3. "Crime and Punishment in Great Britain" por Nigel Walker, (1965) Edinburgh University Press.

4. "Capital Punishment as a deterrent and the alternative" por Gerald Gardiner, Q.C., Gollancz, London.

5. "The Death Penalty in America" an anthology, ed. por Hugo Bedau (1964) Aldine Press, Chicago.

6. "The Homicide Act" por Christopher Hollis (1964) Gollancz, London.

7. "A Calendar of Murder: Criminal Homicide in England since 1957". Terence Morris y Louis Blom-Cooper (1964) Michael Joseph, London.

8. Report of the Royal Commission on Capital Punishment, 1949/1953.. CMD 8932 H.M. Stationery Office, 1953. 